\title{
MODELING OF INTERSTELLAR SURFACE PROCESSES
}

\author{
What does the surface of grains look like?
}

\author{
V. BUCH \\ The Physical Chemistry Department, Hebrew University, \\ Jerusalem, Israel \\ AND \\ J. P. DEVLIN \\ Department of Chemistry, Oklahoma State University, \\ Stillwater, Oklahoma 74078, USA
}

\begin{abstract}
Computational modeling is discussed of some interstellar surface processes. The surface of interstellar grains is envisaged as covered by at least several layers of weakly bonded molecular material. Simulated amorphous ice particles were used to model interaction of such surfaces with gas, including sticking of $\mathrm{H}$ and $\mathrm{D}$ atoms, and adsorption of $\mathrm{H}_{2}$. A possibility of microporosity of interstellar ice mantles is discussed.
\end{abstract}

\section{Introduction}

Computer modeling is by now a standard tool for investigating molecular systems. Its two major varieties include (a) simulations of structure and other thermal properties by solution of statistical mechanics equations for a system of atoms or molecules, and (b) solution of equations of motion for the atoms, in an effort to elucidate mechanisms of molecular processes and to calculate their rates. In our studies, the first variety was used to calculate $\mathrm{H}_{2}$ uptake on amorphous ice, and the second to investigate sticking of $\mathrm{H}$ and $\mathrm{D}$ atoms on interstellar grains. It is well known in surface science that gas - surface interactions are influenced crucially by the structure and the composition of the pertinent solid surfaces. While surface properties of interstellar grains are not known with any degree of certainty, it is suggested here that grains in both dense and diffuse clouds are covered by at least several layers of weakly bonded, rough and disordered molecular material. This is since grains are constantly bombarded by gas, and much of the non- $\mathrm{H}_{2}$ gas component is expected to stick to the surface. While desorption 




Figure 1. A model amorphous ice cluster containing 686 water molecules.

mechanisms must be present, they are not expected to be instantaneous, or $100 \%$ efficient. And although the factors shaping the grain surface are far from being well undersood, low temperature condensation of mixed molecular material is certainly not expected to generate smooth crystalline surfaces.

Computational studies of grain surface processes require a well defined model for such a disordered molecular surface. The model used by us is of an amorphous ice cluster, generated by computer simulation of a process by which amorphous ice is made in the lab, i.e. slow condensation of water vapor (Zhang \& Buch 1990; Buch 1992). Amorphous ice has been used extensively in lab simulations of interstellar spectra and processes, and it appears not unreasonable to use it as a model surface in computer simulations of interstellar surface processes. The model amorphous ice clusters are "grown" on the computer by solving Newton's equations of motion for a sequence of water molecules impinging from a random direction on an ice "nucleus" and sticking to its surface. The initial "nucleus" contains several tens of water molecules; during the course of the simulation, the ice particle is grown to the size of several hundred $\mathrm{H}_{2} \mathrm{O}$; further details can be found in Zhang \& Buch (1990), and Buch (1992). The largest simulated amorphous ice cluster generated by us until now (containing 686 water molecules) is shown in Fig. 1; note its rough and disordered surface. Such amorphous cluster models were used quite successfully in the interpretation of spectroscopy of amorphous ice, and of adsorbate - amorphous ice systems (Buch \& Devlin 1991, 1993; Buch, Silva \& Devlin 1993; Hixson et al. 1992). We describe below some applications to interstellar problems. 


\section{Sticking of $\mathbf{H}$ and $\mathbf{D}$ atoms on interstellar grains}

The sticking probability of hydrogen atoms on grains is of interest, since $\mathrm{H}_{2}$ formation on grains is believed to be preceded by sticking of gaseous atoms to the surface (Duley \& Williams 1984). Thus, the sticking probability is needed to estimate the $\mathrm{H}_{2}$ recombination rates.

The sticking probability was evaluated by solving classical trajectories of $\mathrm{H}$ (or D) atoms impinging on an amorphous ice particle containing 115 water molecules (Buch \& Zhang 1991). An atom was "sent" towards the particle from a random direction, and Newton's equations of motion were solved for the atom and the water molecules of the ice particle. In some of the trajectories, the atom "succeeded" to lose enough energy to the water molecules during the collision, so that it became trapped in the potential well on the grain surface. If in the course of the trajectory, the energy of the atom became negative, the atom was counted as "stuck". The fraction of the trajectories that resulted in sticking was averaged over a thermal distribution of initial conditions for the gas atom (the temperature of the particle was set to $10 \mathrm{~K}$ ). The resulting sticking probability, calculated for the range $T<300 \mathrm{~K}$, could be fit into a simple formula $S_{T}=\left[1+\left(T / T_{0}\right)\right]^{2}$, where $T_{0}=102 \mathrm{~K} / 200 \mathrm{~K}$ for the $\mathrm{H} / \mathrm{D}$ atoms respectively. The surface roughness was seen to influence significantly the collision trajectories, since an atom could be redirected by a collision with one surface "bump" towards another "bump" , thus acquiring additional opportunity to lose energy to the surface. In fact, the sticking trajectories were remarkably long; the atoms scanned a distance of a few tens of angstrom on the surface before losing enough energy to become immobilized. Such long sticking trajectories should enhance surface reactivity of $\mathrm{H}$-atoms impinging on interstellar grains, since an atom may encounter a reactive species on its way. The possibility of surface reactions was not included in the study of Buch \& Zhang, which focused on sticking in the limit of zero coverage.

The above sticking probability values yielded encouraging results in modeling of warm neutral halos around molecular clouds (Andersson \& Wannier 1993). These authors concluded that "the generally accepted rate for $\mathrm{H}_{2}$ formation on grains is too large by a factor of several". The referenced formulas for rates (Hollenbach, Werner \& Salpeter 1971) assumed $S_{T}$ near unity in the relevant temperature range $(<200 \mathrm{~K})$. The agreement with the observations could be reached in that study, after the rates were rescaled by the sticking probability expressions given above.

\section{3. $\mathbf{H}_{2}$ adsorption on amorphous ice}

Recently, Sandord, Allamandola \& Geballe (1993) reported detection of an interstellar infrared band at 2.415 micron, due to condensed $\mathrm{H}_{2}$ in (or on) 
ice mantles of grains. The question arose as to the source of condensed $\mathrm{H}_{2}$. The authors argued that simple adsorption of molecular hydrogen from the gas phase is too inefficient, based on the value of the mean binding energy of $\mathrm{H}_{2}$ on amorphous ice measured in their laboratory. They suggested that $\mathrm{H}_{2}$ is generated photochemically from ice, and trapped in the mantle interior. A possible difficulty of this mechanism is a requirement of a long mantle lifetime, so that a substantial amount of $\mathrm{H}_{2}$ can accumulate inside.

We employed computer simulations to reexamine the adsorption efficiency of $\mathrm{H}_{2}$ on amorphous ice mantles (Buch \& Devlin 1994). While simulations are never perfectly accurate, they enable direct studies of molecular processes under the low pressure interstellar conditions, which is not possible in the lab. The quantum-mechanical Path Integral Monte Carlo (PIMC) technique was employed in this study (Chandler \& Wolynes 1981). PIMC was used to generate snapshots of adsorbate configurations on a model ice particle at a given surface temperature, gas temperature, and gas pressure (Buch \& Devlin 1993). Steady state was assumed for $\mathrm{H}_{2}$, that is, the rate of $\mathrm{H}_{2}$ sticking was assumed equal to the rate of $\mathrm{H}_{2}$ evaporation.

Fig. 2 shows the bare model ice particle, the same particle exposed to $\mathrm{H}_{2}$ under laboratory conditions (gas pressure 0.5 micron $\mathrm{Hg}, T_{\text {gas }}=50 \mathrm{~K}$, $\left.T_{\text {ice }}=12 \mathrm{~K}\right)$, and the same particle under interstellar conditions $(n=8.3$ $\left.10^{4} \mathrm{~cm}^{-3}, T_{\text {gas }}=12 \mathrm{~K}, T_{\text {ice }}=12 \mathrm{~K}\right)$. Under the laboratory conditions, the surface is packed with $\mathrm{H}_{2}$, and the calculated binding energy $(-539 \mathrm{~K})$ is in fact close to the value obtained by Sandford \& Allamandola (1993). Under the low pressure interstellar conditions, there is still a considerable fraction $(\approx 20 \%)$ of $\mathrm{H}_{2}$ adsorbate left on the ice particle, adsorbed in cracks and crevices in the disordered amorphous ice surface; the $\mathrm{H}_{2}$ mean binding energy is now greater by a factor 1.4-1.8 than under laboratory conditions. The above results demonstrate that in the case of highly inhomogeneous surfaces, experimental data obtained under laboratory conditions may not be directly applicable to interstellar conditions. Our estimates suggest that simple adsorption from the gas phase may account for the observed condensed $\mathrm{H}_{2}$ feature; a similar conclusion was reached independently by Dissly, Allen \& Anicich (1994). The advantage of adsorption as a source of condensed $\mathrm{H}_{2}$ (as compared to the proposed photochemical mechanism) is a short time scale required to establish a steady state between adsorbed and gaseous $\mathrm{H}_{2}$ (less than several years). On the other hand, substantial amounts of $\mathrm{H}_{2}$ adsorbate require a high surface to bulk ratio for the interstellar ice mantles. This property has been demonstrated experimentally for the low temperature amorphous ice deposits generated in the laboratory. The question whether the interstellar mantles have a similarly large surface to bulk ratio is far from clear, and is discussed in the next paragraph. 


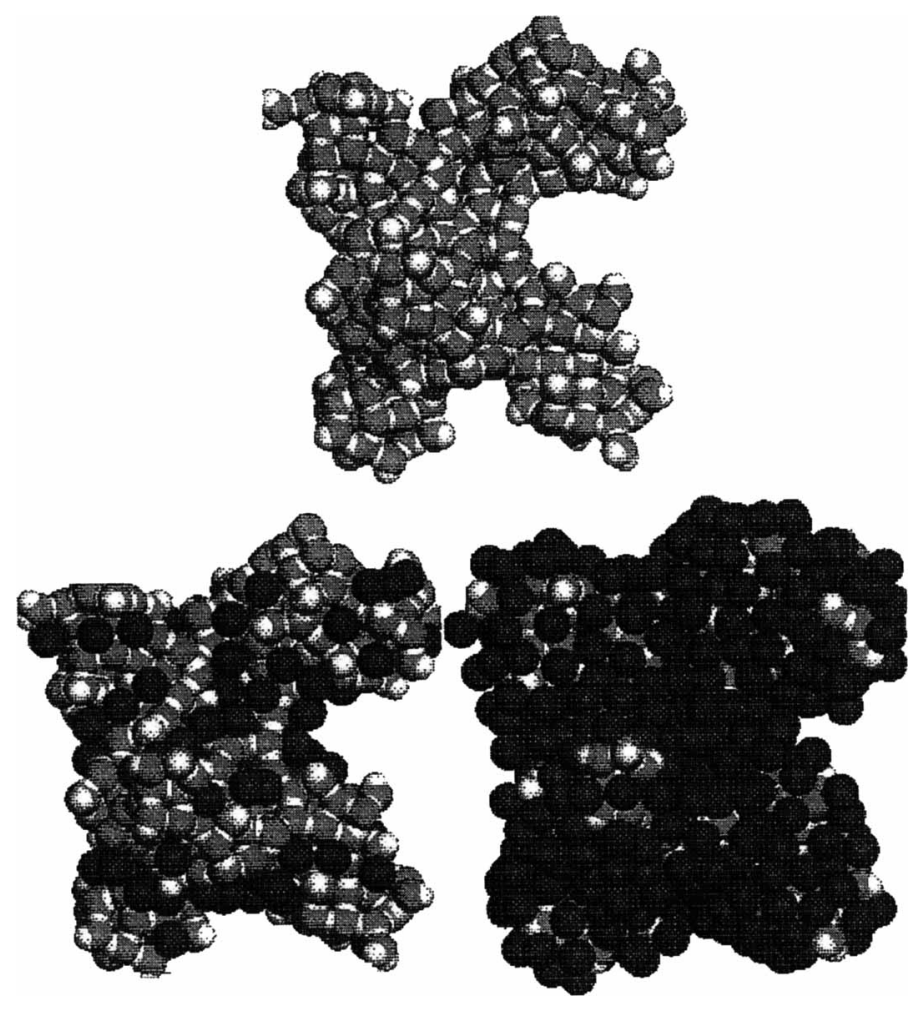

Figure 2. Amorphous ice cluster containing 450 water molecules. Top: bare cluster. Bottom left: with hydrogen adsorbate under interstellar conditions. Bottom right: with hydrogen adsorbate under lab conditions. See also text.

4. Microporosity of laboratory amorphous ice deposits; are interstellar ice mantles microporous?

We first discuss laboratory evidence for microporosity of amorphous ice deposits. It has been demonstrated experimentally (Mayer \& Pletzer 1986, 1987; Pletzer \& Mayer 1989; Schmitt et al. 1987; Rowland \& Devlin 1991; Brown et al. 1996) that slow deposition of $\mathrm{H}_{2} \mathrm{O}$ on a cold substrate $(T<$ $100 \mathrm{~K}$ ) results in an amorphous form of ice of a very large effective surface area with respect to gas adsorption. Analysis of the adsorption isotherms indicated that the material is microporous, i.e., it is pervaded by small cavities (of diameter $<20 \AA$ ) that are accessible from outside to adsorbate gas (Mayer \& Pletzer 1986, 1987; Schmitt et al. 1987).

The physical reasons for this texture of amorphous ice deposits were examined by computer simulations (Buch 1992). Specifically, analysis was 
carried out of the sticking trajectories of water molecules leading to the formation of the model amorphous ice clusters described above (see Fig. 1), and of the resulting cluster surface structure. Due to limitations of computer time, our ice cluster models are too small to be validly considered microporous (the largest linear dimension of the cluster shown in Fig. 1 is 59 angstrom). Still, their surfaces are very rough, and include relatively deep indentations that can be considered an initial stage of the micropore formation. The reasons for this surface roughness were investigated. Formation of such surface texture is due to impaired relaxation of the condensing water molecules at low temperatures. A condensing molecule is attached initially to an available binding site on the surface, that is to a dangling $\mathrm{H}$ or $\mathrm{O}$ atom (i.e., an atom of $\mathrm{H}_{2} \mathrm{O}$ with a missing hydrogen bond). After the attachment, the new molecule "manages" to acquire one more hydrogen bond (or at most two bonds) to neighboring binding sites, but only rarely is its coordination shell completed to 4 during a sticking event. The dangling atoms that provide binding sites for new molecules tend to stick out of the surface. A newly attached molecule sticks out of the surface even more, and can serve as a new binding site, since its coordination shell is not saturated. Moreover protruding binding sites are expected to be more easily accessible to condensing molecules than sites in "deep" locations. This condensation mechanism results in propagation of surface protrusions, and in formation of surface cavities in between.

It should be emphasized that micropores are fragile structures that disappear upon annealing in the 60-120 K range (Mayer \& Pletzer 1986, 1987). Moreover the extent of microporosity of freshly formed amorphous ice deposits is highly sensitive to the experimental preparation conditions; the requirements for obtaining microporous samples include low deposition rate (less than 1 micron / (5 min)), and good thermal contact to the substrate. Still, there is no doubt that slow deposition of thin water films at low $T$ produces microporous amorphous ice.

Are interstellar ice mantles similarly microporous? This possibility, and the corresponding astrophysical implications have been already discussed some 10 years ago by Mayer \& Pletzer (1986). Microporosity would influence both physical and chemical properties of the mantles, such as density, thermal conductivity, interaction with radiation, and surface diffusion and reactivity of adsorbates. Micropores could serve as interesting reaction sites for $\mathrm{H}_{2}$ recombination. Large amounts of non-volatile gas can be stored in the micropores; Mayer \& Pletzer discussed interesting implications on cometary phenomena.

However the formation mechanism of interstellar amorphous ice may be quite different from that in the laboratory. The texture of ice formed by a sequence of surface reaction events (as suggested by a number of kinetic 
models of clouds, e.g. Tielens \& Hagen 1982; d'Hendecourt et al. 1985) is far from clear. Some interstellar factors are expected to favor a fluffy mantle material with cavities. For example, small dust particles accrete on the mantle surface in addition to gas (Draine 1985; Schutte \& and Greenberg 1991), and during this low $T$ accretion many cavities can be reasonably expected to form in the mantle material. Localized impulsive events such as UV and cosmic ray absorption and chemical reactions may both contribute to formation of cavities and their destruction. Moreover $\mathrm{H}_{2}$ adsorbate is expected to accumulate in cavities on the icy surface, and is likely to stabilize the cavities upon further mantle condensation (experiments of Dissly, Allen \& Anicich (1994) on condensation of 1:36 $\mathrm{H}_{2} \mathrm{O} / \mathrm{H}_{2}$ mixtures seem in accord with this picture).

While the question of mantle microporosity is far from clear, it can be investigated by infrared observations (Buch \& Devlin 1994; McCoustra \& Williams 1996). Spectroscopic studies of amorphous ice revealed two satellite features to the blue from the main 3.07 micron $\mathrm{OH}$ stretch band, at 2.706 and 2.690 micron (3696 and $3718 \mathrm{~cm}^{-1}$, Rowland \& Devlin 1991; Rowland, Fisher \& Devlin 1991). With the help of simulations (Buch \& Devlin 1991), the two features were identified as characteristic of the micropore surface in amorphous ice. The two bands are due to dangling $\mathrm{OH}$ bonds (i.e. $\mathrm{OH}$ bonds of $\mathrm{H}_{2} \mathrm{O}$ in which the $\mathrm{H}$ atom is not involved in a hydrogen bond) which stick out of the micropore surface. The 2.706 (2.690) micron bands are due to dangling $\mathrm{OH}$ of 2 (3) coordinated surface $\mathrm{H}_{2} \mathrm{O}$ molecules, respectively. The 2.706 micron feature corresponds to a highly unannealed and very microporous form of ice, and disappears upon annealing to $\approx 60 \mathrm{~K}$. The 2.690 micron feature persists in the spectra as long as the material has enough surface to make it visible. Upon exposure to adsorbates, the bands are shifted in an adsorbate specific manner, confirming the surface assignment. It should be noted that the ability to penetrate the amorphous ice micropores and coat their surfaces is adsorbate dependent and temperature specific, the measured thresholds for penetration are $18 \mathrm{~K}$ for $\mathrm{N}_{2}, 26 \mathrm{~K}$ for $\mathrm{CO}$ and $34 \mathrm{~K}$ for $\mathrm{CH}_{4}$ (Devlin 1992). $\mathrm{H}_{2}$ was found to penetrate the pores at temperatures as low as $12 \mathrm{~K}$. Larger molecules such as benzene do not penetrate the micropores from outside, but can be introduced into amorphous ice by codeposition.

If the dangling $\mathrm{OH}$ infrared bands are observed in space, they would provide strong evidence for microporosity. A possible complication is a frequency shift due to adsorbates (Rowland, Fisher \& Devlin 1991; Devlin \& Buch 1995); if the micropore surface is covered by a weakly bonded adsorbate, the dangling $\mathrm{OH}$ bands will be still visible, but at different (typically lower) frequencies. Some frequency shift values are $-14 \mathrm{~cm}^{-1}$ for $\mathrm{H}_{2}$ adsorbate, $-22 \mathrm{~cm}^{-1}\left(\mathrm{~N}_{2}, \mathrm{CH}_{4}\right)$, and $-60 \mathrm{~cm}^{-1}(\mathrm{CO})$. Thus, if the micropores 
in fact exist in the mantles and if they are covered by one dominant kind of non-polar adsorbate, the dangling $\mathrm{OH}$ band may be still visible, but a multicomponent adsorbate layer may broaden the (weak) bands to the point of being unobservable. Moreover strongly hydrogen bonding adsorbates would shift the dangling $\mathrm{OH}$ frequency to the range of the main (bulk ice) $\mathrm{OH}$ stretch band. It is not clear to us if the interstellar micropores should be covered by adsorbate.

\section{Low T amorphous ice deposits: a low density or a high density material?}

The last comment pertains to possible confusion in current literature concerning the density of the low temperature amorphous ice deposits, as measured in the laboratory. The question discussed here is whether low $T$ amorphous ice deposits constitute a low density or a high density material. Of course the density value depends on whether the micropores are included in the volume or excluded from it. For example, recent optical interference studies of amorphous ice films (Brown et al. 1996), suggest low density values in the range $0.6-0.8 \mathrm{~g} / \mathrm{cm}^{3}$ for $20-90 \mathrm{~K}$ deposits (in these measurements the micropores pervading the volume of the film are included in the volume). On the other hand, Jenniskens et al. (1995) suggested based on their electron diffraction studies, that deposition at $T<30 \mathrm{~K}$ yields a high density form of amorphous ice, apparently of density near $1.17 \mathrm{~g} / \mathrm{cm}^{3}$. The possibility that much of the volume of this material may be empty was not considered by these authors.

We suggest that the correct description of the low $T$ amorphous ice deposits is "highly inhomogeneous" rather than either low or high density, since in the microporous deposits, the compact ice material is interspersed by voids. The diffraction studies measure correlations between neighboring molecules, and thus are sensitive to the "compact" component of amorphous ice. Our computational studies are in qualitative accord with the notion that this compact component has in fact a density higher than $1 \mathrm{~g} / \mathrm{cm}^{3}$ (Zhang \& Buch 1990). This is since condensation of $\mathrm{H}_{2} \mathrm{O}$ is associated with formation of molecular many-ring structures which are smaller than the 6-membered rings characteristic of hexagonal ice.

Still, the radial distribution functions generated in our simulations of condensation (Zhang \& Buch 1990), and also in previous diffraction studies of amorphous ice deposits are quite different from those presented by Jenniskens et al. (see their Fig. 2), in that in the latter study the first and the second diffraction peaks merge rather than being well separated. Since the latter behavior is characteristic of ice "damaged" by fast electrons or high pressure, it appears to us that their amorphous ice was affected by electron 
bombardment. It would be of interest to interstellar chemists to find out how different types of processing (UV radiation, fast particles) affect the extent of microporosity of amorphous ice, and the effective surface area with respect to gas adsorption.

\section{References}

Andersson, B. G., Wannier, P. G. 1993, ApJ, 402, 585

Brown, D. E., George, S. M., Huang, C., Wong, E. K. L., Rider, K. B., Smith, R. S., Kay, B. D., 1996, J. Phys. Chem., 100, 4988

Buch, V. 1992, J. Chem. Phys., 96, 3814

Buch, V., Devlin, J. P. 1991, J. Chem. Phys., 94, 4091

Buch, V., Devlin, J. P. 1993, J. Chem. Phys., 98, 4195

Buch, V., Devlin, J. P. 1994, ApJ, 431, L135

Buch, V., Silva, S. C., Devlin, J. P. 1993, J. Chem. Phys., 99, 2265

Buch, V., Zhang, Q. 1991, ApJ 379, 647

Chandler, D., Wolynes, P. G. 1981, J. Chem. Phys., 74, 4078

d' Hendecourt, L. B., Allamandola, L. J., Greenberg, J. M., 1985, A\&A 152, 130

Devlin, J. P. 1992, J. Phys. Chem., 96, 6185

Devlin, J. P., Buch, V. 1995, J. Phys. Chem., 99, 16534

Dissly, R. W., Allen, M. Anicich, V. G. 1994, ApJ, 435, 685

Draine, B. T., 1985, in Protostars and Planets II, ed. D Black and M. Mathews (Tucson: University of Arizona Press), p. 621

Duley, W. W., Williams, D. A. 1984, Interstellar Chemistry (London: Academic)

Hixson, H. G., Wojcik, M. J., Devlin, M. S., Devlin, J. P., Buch, V. 1992, J. Chem. Phys., 97,753

Hollenbach, D. J., Werner, M. W., Salpeter, E. E. 1971, ApJ, 163, 165

Jenniskens, P., Blake, D. F., Wilson, M. A., Pohorille, A. 1995, ApJ 455, 389

Mayer, E., Pletzer, R. 1986, Nature 319, 298

Mayer, E., Pletzer, R. 1987, Journal de Physique C1, 581

McCoustra, M., Williams, D.A. 1996, M.N.R.A.S. 279, L53

Pletzer, R., Mayer, E., 1989, J. Chem. Phys. 90, 5207

Rowland, B., Devlin, J. P. 1991, J. Chem. Phys., 94, 812

Rowland, B., Fisher, M., Devlin, J. P. 1991, J. Chem. Phys., 95, 1378

Sandford, S. A., Allamandola, L. J. 1993, ApJ, 409, L65

Sandford, S. A., Allamandola, L. J., Geballe, T. R., 1993, Science 262, 400

Schmitt, B., Ocampo, J., Klinger, J., 1987, J. Phys. (Paris) 48, C1-519

Schutte, W. A., Greenberg, J. M., 1991, A\&A 244, 190

Tielens, A. G. G. M., Hagen, W., 1982, A\&A 114, 245

Zhang, Q., Buch, V. 1990, J. Chem. Phys., 92, 5004 


\section{Discussion}

Langhoff: Have you used simulated annealing techniques to vary the porosity of your ices? Electrical conductivity is a sensitive measure of porosity - can this be determined or used to advantage?

Buch: My ability to represent porosity is limited by the size of the simulated ice models, but yes, within this limitation I did investigate collapse of surface indentations and cavities (which look like beginnings of pores) upon simulated heating of model low temperature condensates (Buch 1990, J. Chem. Phys. 93, 2631). I am afraid I do not know enough about electrical conductivity of amorphous ice; experimental quantities to which we related in simulations have been predominantly diffraction patterns, and vibrational and rotational spectra of amorphous ice and of molecular adsorbates.

Pecker: In HII regions (full of ionizing radiation) one observes grains. We would like to know the sticking probabilities of $\mathrm{H}^{+}$, i.e., protons. What can you say about these probabilities?

Buch: My guess is that the sticking probability is of order unity, because of $\mathrm{H}^{+}$ reacting chemically with grain surfaces. But I have never really thought about it. Very interesting question.

Eberhardt: How much D-fractionation would you expect in the surface layer? Buch: Tielens investigated this question in the eighties. According to his calculations - a lot. 